\title{
Review Article \\ Xerostomia, Hyposalivation, and Salivary Flow in Diabetes Patients
}

\author{
Rosa María López-Pintor, Elisabeth Casañas, José González-Serrano, Julia Serrano, \\ Lucía Ramírez, Lorenzo de Arriba, and Gonzalo Hernández
}

Department of Oral Medicine and Surgery, School of Dentistry, Complutense University, 28040 Madrid, Spain

Correspondence should be addressed to Rosa María López-Pintor; rosamlpm@yahoo.es

Received 15 April 2016; Accepted 31 May 2016

Academic Editor: Rafael A. Delgado-Ruiz

Copyright (C) 2016 Rosa María López-Pintor et al. This is an open access article distributed under the Creative Commons Attribution License, which permits unrestricted use, distribution, and reproduction in any medium, provided the original work is properly cited.

\begin{abstract}
The presence of xerostomia and hyposalivation is frequent among diabetes mellitus (DM) patients. It is not clear if the presence of xerostomia and hyposalivation is greater in DM than non-DM patients. The aims of this systematic review are (1) to compare the prevalence rates of xerostomia, (2) to evaluate the salivary flow rate, and (3) to compare the prevalence rates of hyposalivation in DM versus non-DM population. This systematic review was conducted according to the PRISMA group guidelines by performing systematic literature searches in biomedical databases from 1970 until January 18th, 2016. All studies showed higher prevalence of xerostomia in DM patients in relation to non-DM population, $12.5 \%-53.5 \%$ versus $0-30 \%$. Studies that analyzed the quantity of saliva in DM population in relation to non-DM patients reported higher flow rates in non-DM than in DM patients. The variation flow rate among different studies in each group (DM/CG) is very large. Only one existing study showed higher hyposalivation prevalence in DM than non-DM patients (45\% versus $2.5 \%$ ). In addition, quality assessment showed the low quality of the existing studies. We recommend new studies that use more precise and current definitions concerning the determination and diagnosis of DM patients and salivary flow collection.
\end{abstract}

\section{Introduction}

Diabetes mellitus $(\mathrm{DM})$ is an endocrine disease characterized by a deficit in the production of insulin with consequent alteration of the process of assimilation, metabolism, and balance of blood glucose concentration. DM has become a worldwide public health problem. In recent years, the global prevalence of DM has increased substantially, reaching $8.3 \%$ in 2014, which corresponds to 387 million patients [1]. Essentially, there are two types of DM: type 1 DM (T1DM) and type $2 \mathrm{DM}$ (T2DM). T1DM accounts for approximately $5 \%$ of diagnosed diabetes cases [2].

Xerostomia is a subjective complaint of dry mouth, whereas hyposalivation is an objective decreased of salivary flow. The clinical method most often employed for the diagnosis of salivary dysfunction is a sialometry test. Hyposalivation is considered to appear when salivary flow rates are under $0.1 \mathrm{~mL} / \mathrm{min}$ at rest (UWS) or $0.7 \mathrm{~mL} / \mathrm{min}$ under stimulation (SWS). Xerostomia is often associated with hyposalivation, but not always. And many cases of xerostomia have been described in patients with a normal salivary flow rate [3-6].

Several factors are capable of inducing salivary disorders in DM patients such as ageing, head and neck radiotherapy, systemic disorders, and several drugs [5]. Systemic diseases associated with xerostomia include rheumatologic chronic inflammatory disorders (Sjögren syndrome, rheumatoid arthritis, and systemic lupus erythematosus), endocrine disorders (DM, hyperthyroidism, and hypothyroidism), neurologic disorders (depression and Parkinson's disease), genetic disorders, metabolic disorders (dehydration, bulimia, anaemia, and alcohol abuse), infectious disorders (HIV/AIDS, HCV infection), and others (fibromyalgia, graftversus-host-disease, sarcoidosis, and chronic pancreatitis). Many cases of xerostomia are also related to psychological conditions like depression and anxiety $[5,6]$.

Both types of DM, T1DM and T2DM, have been associated previously with xerostomia [7-12]. There are also studies 
that have showed a decreased salivary flow in DM patients in relation to non-DM patients [7, 8, 12-21]. The reason for these problems could be due to damage to the gland parenchyma, alterations in the microcirculation to the salivary glands, dehydration, and disturbances in glycemic control [5].

Considerable debate exists surrounding the issue, if the presence of xerostomia and hyposalivation is greater in DM than non-DM patients. No systematic review has been performed up to now. Given the lack of systematic knowledge, we have conducted the first systematic review concerning the prevalence of xerostomia and hyposalivation in DM (compared to non-DM) patients. We also have analyzed the differences in the rate of salivary flow between DM and nonDM patients.

The main objectives of this review were (1) to compare the prevalence rates of xerostomia in the DM and non-DM population, (2) to evaluate the salivary flow rate in the DM and non-DM population, and (3) to compare the prevalence rates of hyposalivation in the DM and non-DM population.

\section{Materials and Methods}

The systematic review was performed according to the PRISMA (Preferred Reporting Items for Systematic Reviews and Meta-Analyses) guidelines [23].

2.1. Focused Question. Based on the PRISMA guidelines, 3 focused questions were constructed. The addressed focused questions (PICO) were as follows: (1) Do DM patients have higher xerostomia prevalence than non-DM patients? (2) Is the salivary flow rate lower in DM patients compared to nonDM patients? (3) Do DM patients have higher hyposalivation prevalence than non-DM patients?

2.2. Search Strategy. A comprehensive literature search was conducted by searching the international biomedical literature databases. PubMed/MEDLINE (National Library of Medicine, Bethesda, Maryland), Scopus, and Cochrane database were searched from 1970 until January 18th, 2016, using different combinations of the following keywords: diabetes; xerostomia; dry mouth; hyposalivation; and salivary flow. Moreover, we performed an additional handsearch to find potential eligible studies as reference lists of review articles and relevant studies.

\subsection{Study Selection}

2.3.1. Inclusion Criteria. Full-text articles were included if they met the inclusion criteria with respect to types of studies, types of population, and the main outcome/s regardless of the time period of study and the year of publication.

Types of Studies. The studies had to be (1) original studies, (2) cross-sectional studies, (3) comparative studies (DM group and healthy control group (CG)), and (4) only in humans. As we evaluated prevalence rates review articles, experimental studies, longitudinal studies, case-reports, commentaries, and Letters to the Editor were excluded. We did not include unpublished articles.

Types of Population. Individuals with diabetes could have T1DM or T2DM. We also considered other diabetes classifications, namely, insulin-dependent (IDDM) and non-insulindependent DM (NIDDM). The total population with DM did not have to suffer specific diseases apart from DM (e.g., end-stage renal disease and hypertension). Individuals without DM were also considered with the aim of comparing prevalence and flow rates between the DM and non-DM population. Individuals without DM did not have to have specific diseases.

Outcomes. The definitions of xerostomia, quantity of salivary flow rate, and hyposalivation are detailed below. Different questions to assess xerostomia were considered: Does your mouth feel dry frequently? Does your mouth usually feel dry, especially during meals? Does your mouth feel dry when you are eating a meal? Do you have difficulties swallowing foods if you eat without additional fluids? Positive response to one of these questions and the consideration of patient's subjective feeling of dry mouth were considered to be xerostomia. Different types of salivary flow rate were considered: UWS (nonstimulated salivary flow), SWS (stimulated salivary flow), USP (nonstimulated parotid flow), SSP (stimulated parotid flow), and SSS (stimulated submandibular/sublingual flow). Furthermore, hyposalivation was considered when UWS $<$ $0.1 \mathrm{~mL} / \mathrm{min}$ or SWS $<0.7 \mathrm{~mL} / \mathrm{min}$, but we included studies that considered hyposalivation when UWS $<0.3 \mathrm{~mL} / \mathrm{min}$ and SWS $<0.5 \mathrm{~mL} / \mathrm{min}$. The main outcomes were the prevalence of xerostomia and/or hyposalivation in percentage and/or the quantity of salivary flow rate in $\mathrm{mL} / \mathrm{min}$.

2.3.2. Exclusion Criteria. Studies were excluded if they were published in a language other than English. They were also excluded if they solely reported prevalence of xerostomia/hyposalivation and salivary flow rates among persons with DM in relation to the total population (DM and non$\mathrm{DM}$ ) and not exclusively to the diabetic (possibly compared to the non-DM) population.

2.4. Data Collection and Extraction. Two authors (Rosa María López-Pintor and Elisabeth Casañas) independently screened all the retrieved titles and abstracts identified through the search strategies to identify potentially eligible articles. Full texts of relevant studies judged by title and abstract were read and independently assessed with reference to the eligibility criteria by two authors (Rosa María LópezPintor and José González-Serrano). Disagreements were resolved by discussion with a third reviewer (Julia Serrano). Data extraction was performed including information about first author, publication year, country, study population, mean age, type of DM, DM diagnosis (if available), definition of xerostomia, definition of hyposalivation (if available), type of flow rate, and data sources of the study. With regard to the results, xerostomia prevalence (\%) and salivary flow rate $(\mathrm{mL} / \mathrm{min})$, as well as hyposalivation prevalence (\%) of DM 
TABLE 1: JBI critical appraisal checklist for studies reporting prevalence data.

\begin{tabular}{|c|c|c|c|c|}
\hline \multirow{2}{*}{\multicolumn{5}{|c|}{$\begin{array}{l}\text { Assessment items } \\
\text { (1) Was the sample representative of the target population? }\end{array}$}} \\
\hline & & & & \\
\hline (2) Were study participants recruited in an appropriate way? & & & & \\
\hline (3) Was the sample size adequate? & & & & \\
\hline (4) Were the study subjects and the setting described in detail? & & & & \\
\hline (5) Was the data analysis conducted with sufficient coverage of the identified sample? & & & & \\
\hline (6) Were objective, standard criteria used for the measurement of the condition? & & & & \\
\hline (7) Was the condition measured reliably? & & & & \\
\hline (8) Was there appropriate statistical analysis? & & & & \\
\hline (9) Are all important confounding factors/subgroups/differences identified and accounted for? & & & & \\
\hline (10) Were subpopulations identified using objective criteria & & & & \\
\hline
\end{tabular}

and non-DM groups, were extracted. The reported statistical signification was extracted if it was available.

2.5. Quality Assessment. In the final selection of eligible studies, we assessed features that could potentially bias the estimates of xerostomia/flow rate/hyposalivation using the Joanna Briggs Institute Prevalence Critical Appraisal Tool (Table 1) [24]. Using this tool we defined criteria based on clinical and epidemiological expertise and ranked potential sources of bias into low or high risk of bias. Scores of $0-$ 5 were evaluated as "low quality" while those of 5-10 were considered to indicate "high quality."

Critical appraisal was conducted by two reviewers (Gonzalo Hernández and Lucía Ramírez) independently of each other. The reviewers met to discuss the results of their critical appraisal; if the two reviewers disagreed on the final critical appraisal and could not be resolved through discussion, a third reviewer (Julia Serrano) was required.

2.6. Categorization of Studies. Due to the high heterogeneity of the studies, we analyzed the outcomes of interest in accordance with the prevalence of xerostomia or salivary quantity flow rate/hyposalivation (if available), type of DM, and age (adults $\geq 19$ years old/children and adolescents). There were studies that reported xerostomia prevalence and flow rate; therefore, there could be two groups. The following categories were the result: (1) xerostomia studies in adults T2DM, (2) xerostomia studies in adults NIDDM, (3) xerostomia studies in children and adolescents T1DM, (4) salivary flow rate studies in adults T1DM, (5) salivary flow rate studies in adults IDDM, (6) salivary flow rate/hyposalivation prevalence studies in adults T2DM, (7) salivary flow rate/hyposalivation prevalence studies in children and adolescents T1DM, and (8) salivary flow rate/hyposalivation prevalence studies in children and adolescents IDDM.

2.7. Statistic Methods. The results of xerostomia prevalence from the included studies were presented as a percentage. The results of quantity salivary flow rate were presented as mean \pm standard deviation (if available). Hyposalivation prevalence results were shown as a percentage. The age of different populations was presented as mean \pm standard deviation, but there were studies that categorized the age or presented only the mean. We showed the possible statistical signification if it was available.

Due to heterogeneity of results, we did not perform a meta-analysis.

\section{Results}

3.1. Searching and Inclusion. The initial search yielded 53 studies. Thirty-eight studies, which did not fulfill the eligibility criteria, were excluded (the Appendix). A total of 15 articles were included and processed for data extraction. The selection procedure is presented in Figure 1.

3.2. Study Design and Quality Assessment. With regard to the main outcome, 7 papers considered xerostomia prevalence (Table 2), and 12 articles considered quantity of salivary flow rate in DM patients (Table 3 ), while 4 papers considered both. Only one paper about salivary flow rate in DM population considered hyposalivation prevalence as outcome (Table 3 ). The results are presented in two parts, xerostomia studies and salivary flow rate/hyposalivation studies.

3.2.1. Xerostomia Studies. We found 7 studies about xerostomia prevalence that met our inclusion criteria. Two of them, written by Sandberg et al. $[9,10]$, presented the same study population. Therefore, we considered these two studies as one study in Table 2. The majority of studies that reported prevalence of xerostomia in DM patients were performed in adults $(n=6), 5$ studies in T2DM patients and one in NIDDM. Only one study was performed in children and adolescents T1DM. One study carried out in adults T2DM [18] did not show xerostomia prevalence rates, but it was included due to presence in the results of explanation of no significant correlation in xerostomia in DM/CG patients.

With respect to the recruitment of patients, three studies had selected their DM patients from an endocrinology service or a diabetic care unit of a specialized medical care or hospital, two from a geriatric center and one (the two studies realized by Sanberg et al. $[9,10]$ with the same population) had sourced the DM patients from a register of primary health care. Control patients were selected from oral health centers $(n=4)$ and geriatric centers $(n=2)$. 


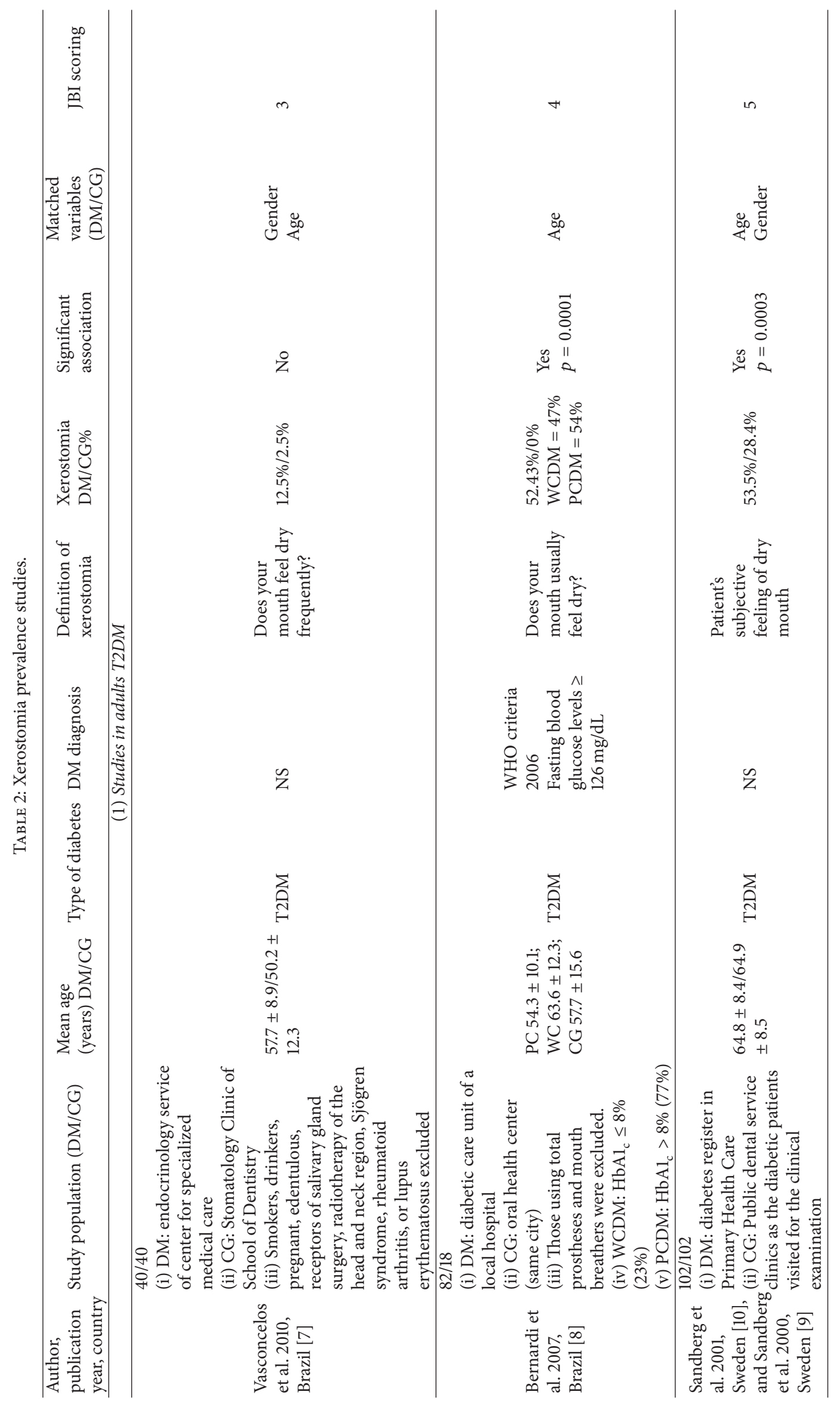




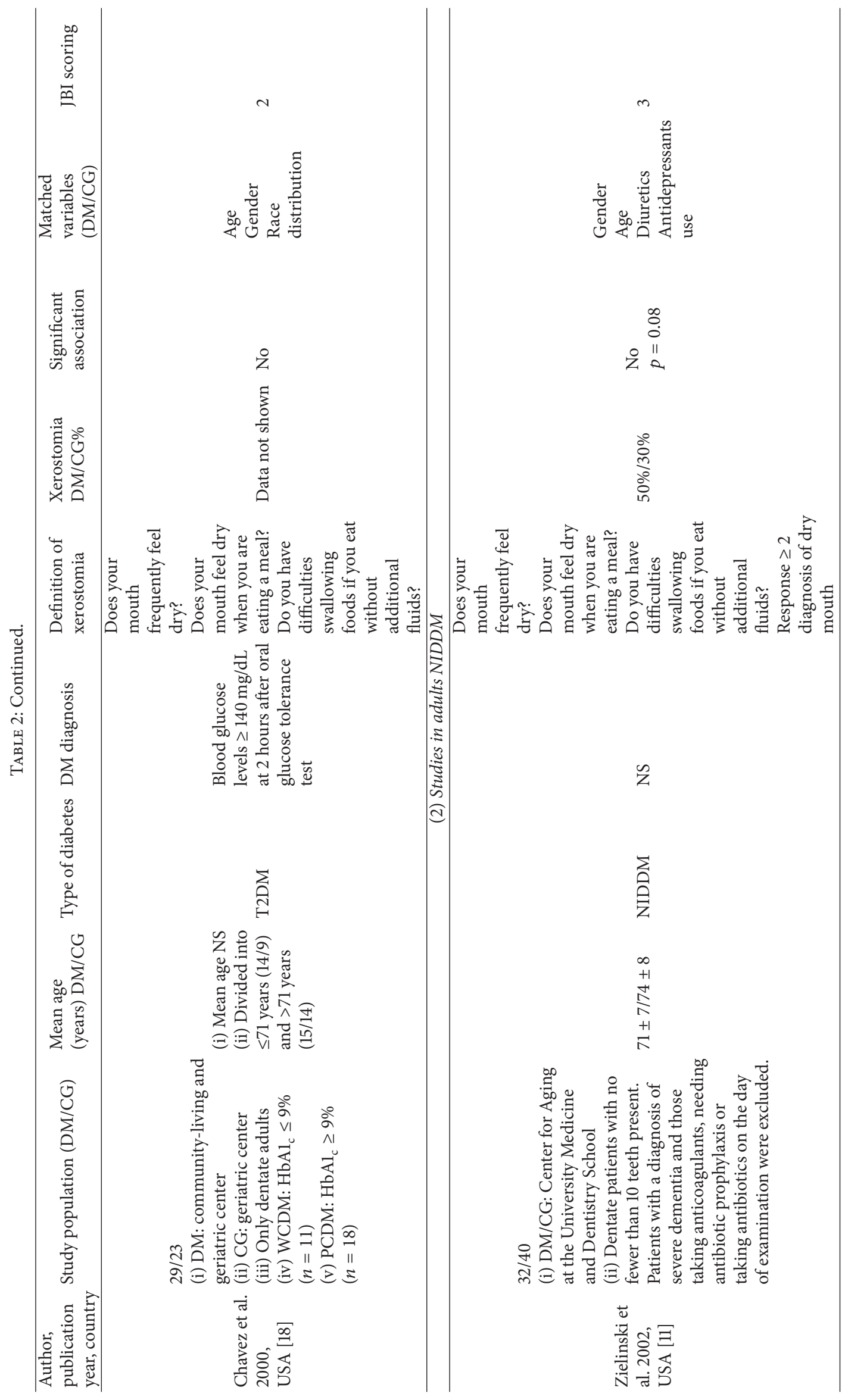




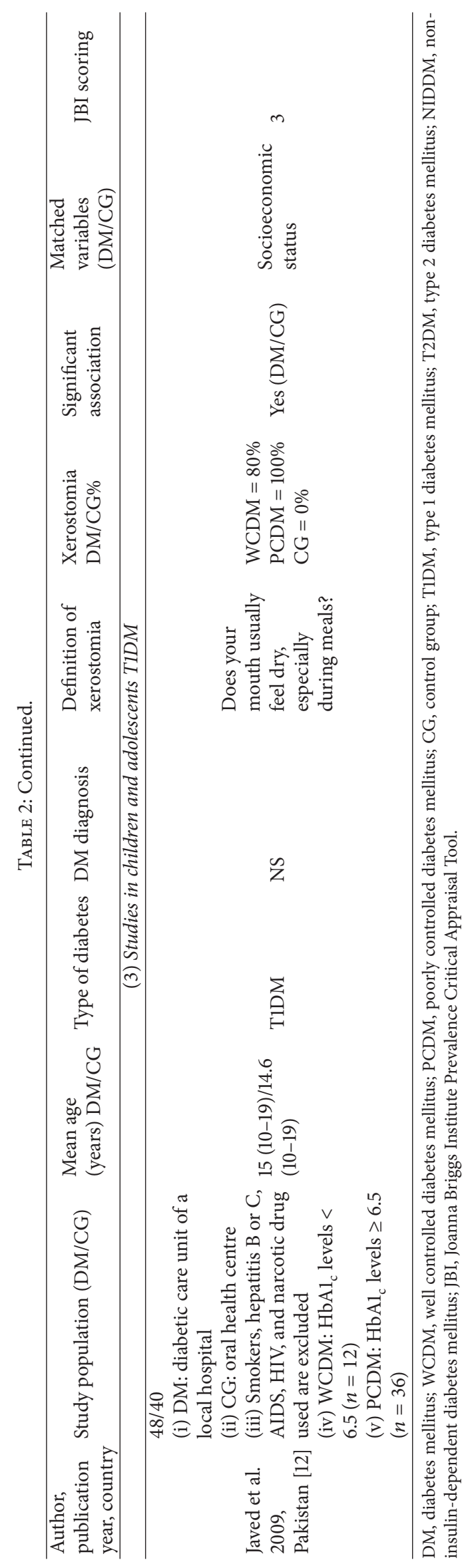




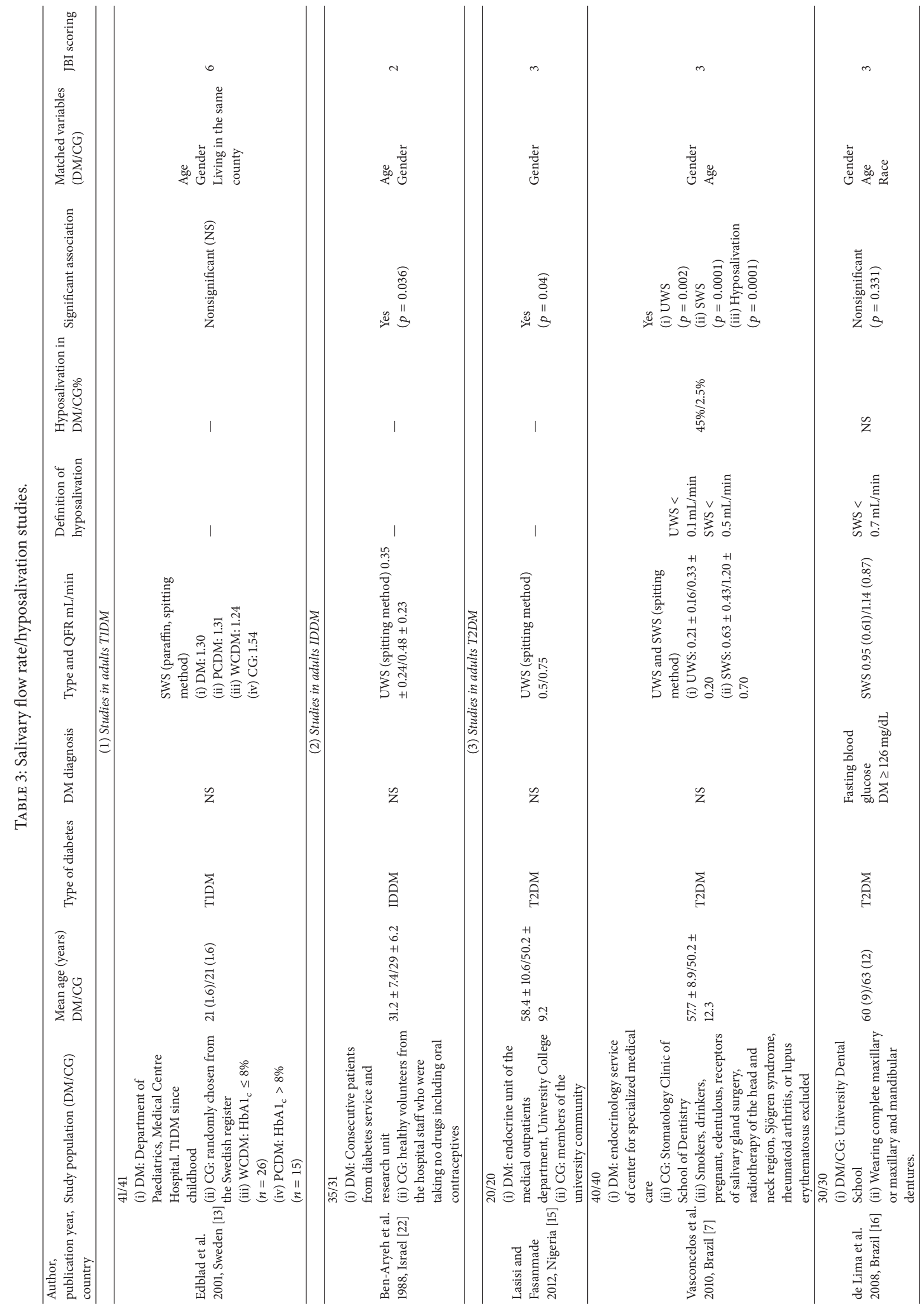




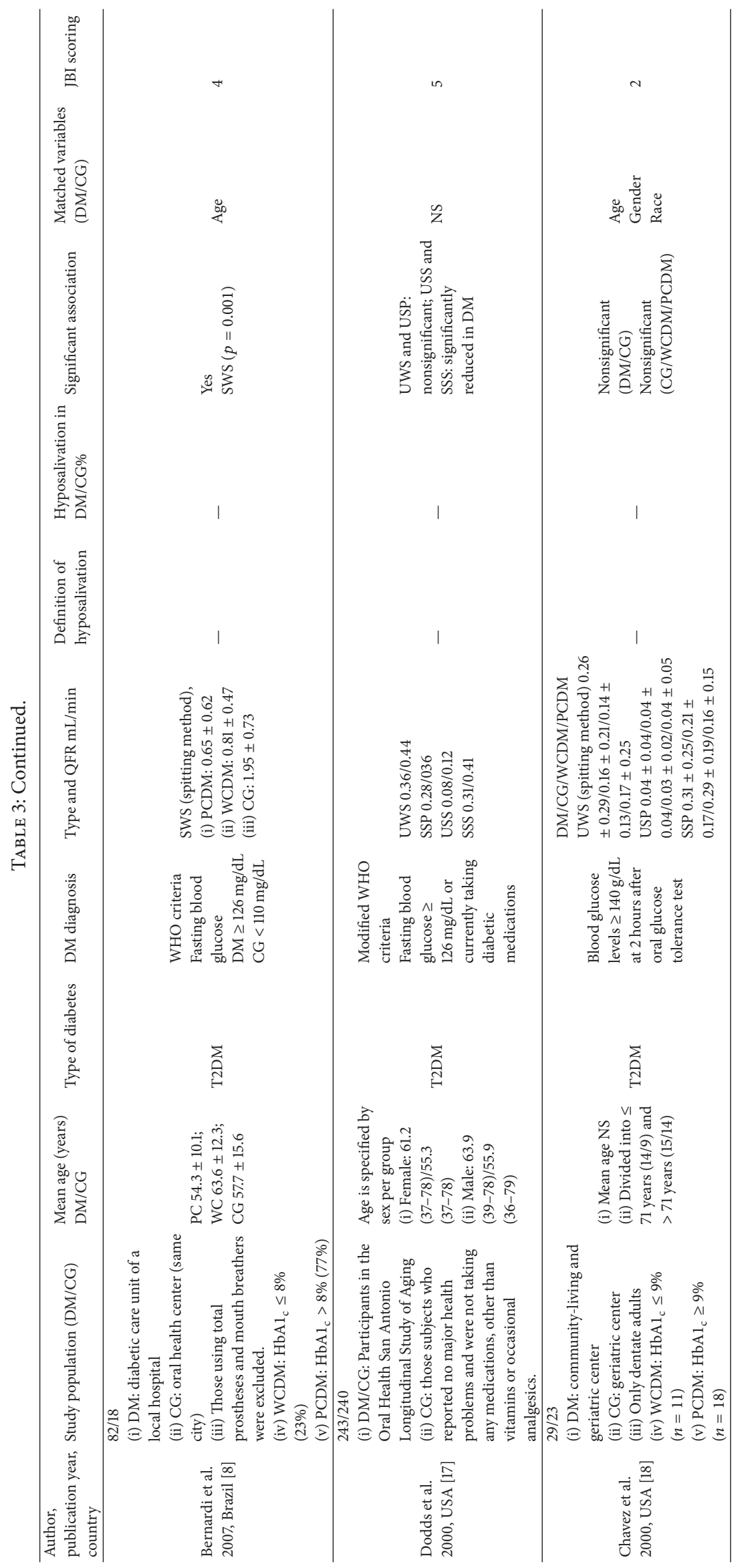




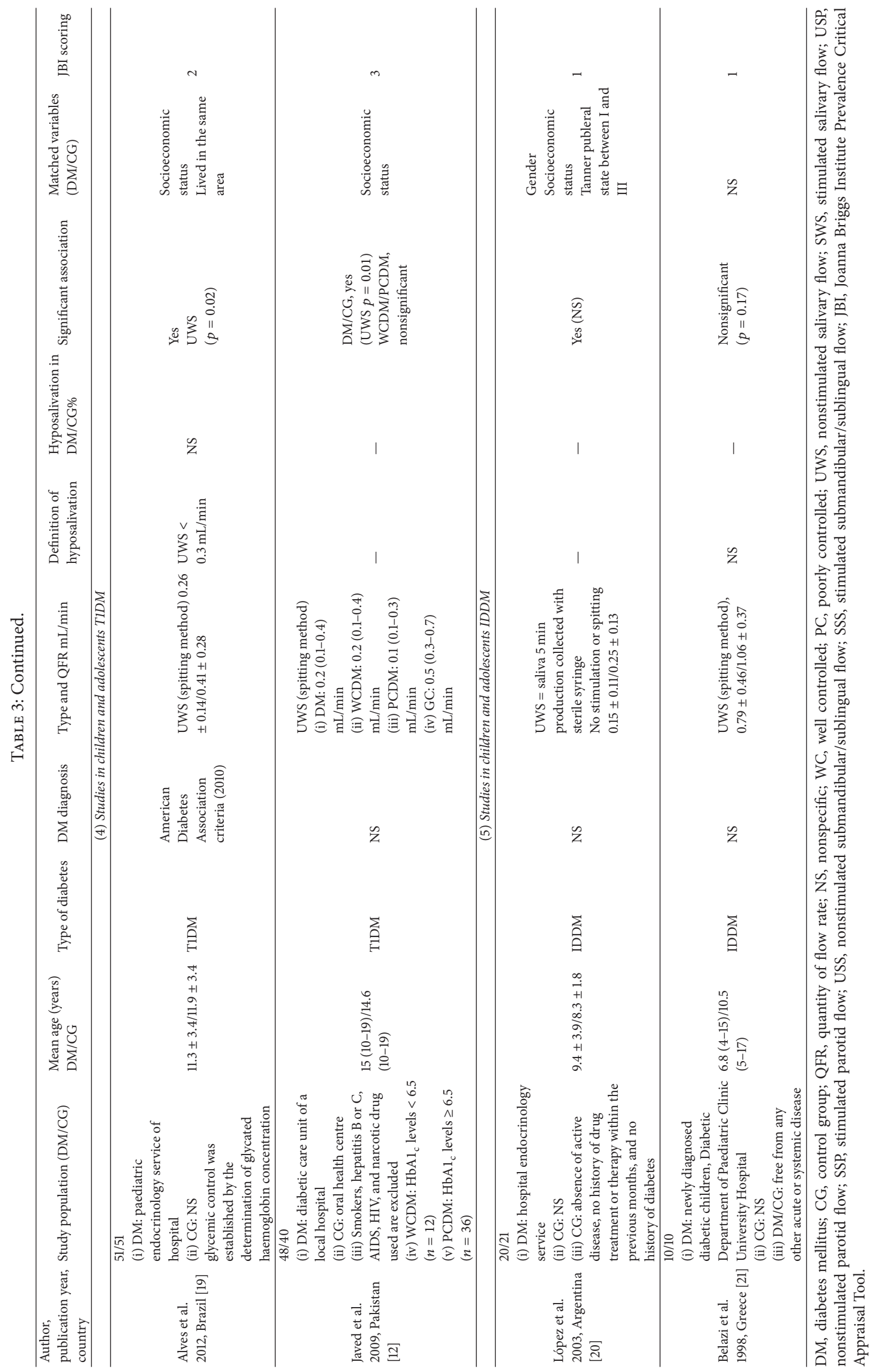




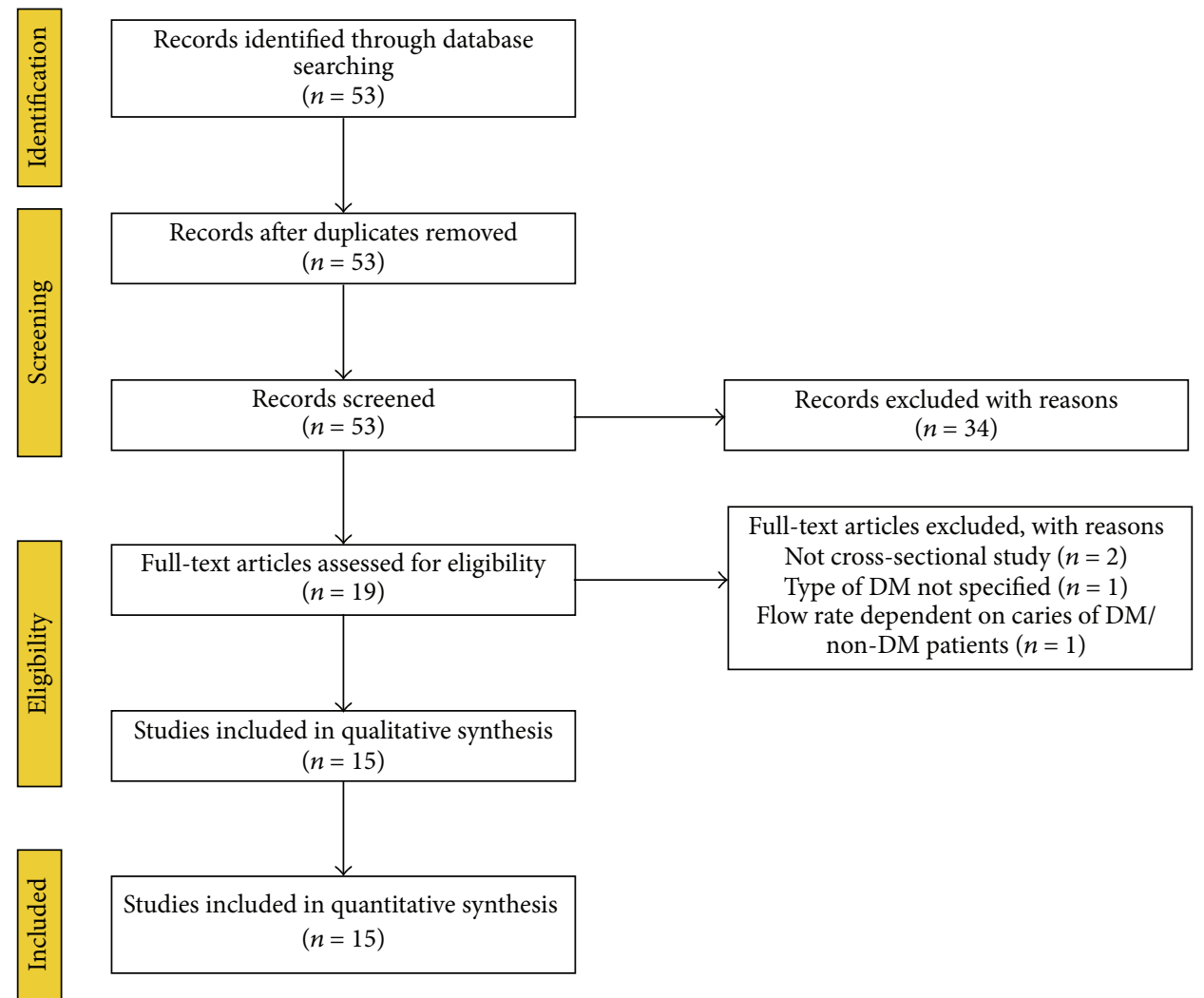

Figure 1: Flowchart of the systematic review process.

The studies included a minimum of 29 and a maximum of 102 DM patients and 18-102 control patients. Only two studies specified the DM diagnosis, one WHO criteria 2006 (fasting blood glucose greater $\geq 126 \mathrm{mg} / \mathrm{dL}$ ) and another one blood glucose levels $\geq 140 \mathrm{mg} / \mathrm{dL}$ at 2 hours after oral glucose tolerance test. No one study reported duration of DM and three studies $[8,12,18]$ reported the $H b A 1_{c}$ levels and classified the patients in well controlled DM (WCDM) and poorly controlled DM (PCDM).

DM and CG participants were matched by gender in 4 studies, by age in 5 studies, by race distribution in one, by diuretics and antidepressants treatment in one, and by socioeconomic status in another one. With regard to statistical significance, three studies $[8-10,12]$ found that DM patients had more significant xerostomia prevalence than non-DM patients. Only one study [18] did not realize the appropriate statistical methods.

Regarding quality assessment all studies obtained scores $\leq 5$; therefore the studies were evaluated as "low quality" (Table 2). Due to the poor quality of the included studies no meta-analysis was performed.

3.2.2. Salivary Flow Rate/Hyposalivation Studies. We found 12 studies about quantity of salivary flow rate that met our inclusion criteria; one of them considered hyposalivation prevalence as outcome (Table 3 ). The majority of studies were carried out in adults $(n=8), 6$ studies in T2DM patients, one in T1DM patients, and another one in IDDM. Four studies were carried out in children and adolescents, 2 in T1DM patients and 2 in IDDM.

Three studies recruited their DM patients from a diabetes care unit of a hospital, 3 from an endocrine unit, 3 from a pediatric endocrinology service, one from a university dental school, one from an oral health study, and another one from community-living/geriatric centers. Non-DM patients came from varied origins: oral health centers $(n=3)$, Swedish register $(n=1)$, healthy volunteers from a hospital staff $(n=1)$, members of a university community $(n=1)$, patients of a university dental school $(n=2)$, and participants in an oral health study of aging $(n=1)$, and 3 studies did not specify the origin. The studies included a minimum of 10 and a maximum of $243 \mathrm{DM}$ patients and a minimum of 10 and a maximum of 240 non-DM patients.

Five studies specified the DM diagnosis, two WHO criteria 2006 (fasting blood glucose $\geq 126 \mathrm{mg} / \mathrm{dL}$ ), one modified WHO criteria 2006 (fasting blood glucose $\geq 126 \mathrm{mg} / \mathrm{dL}$ ) or currently taking diabetic medications, one blood glucose levels $\geq 140 \mathrm{mg} / \mathrm{dL}$ at 2 hours after oral glucose tolerance test, and the last one American Diabetes Association criteria 2010 $\left(\mathrm{HbA} 1_{\mathrm{c}}\right.$ levels $\geq 6.5 \%$ or fasting blood glucose $\left.\geq 126 \mathrm{mg} / \mathrm{dL}\right)$. One study [13] reported that DM patients suffered T1DM since childhood, and there was another study [21] that only included newly diagnosed diabetic children. With respect to dental condition, one study [7] did not include edentulous patients, one study [16] recruited only patients wearing 
complete maxillary or maxillary and mandibular dentures, and another one [8] excluded patients using total prostheses and mouth breathers. Four studies $[8,12,13,18]$ reported the $\mathrm{HbAl}_{\mathrm{c}}$ levels and classified the patients in WCDM and PCDM.

$\mathrm{DM}$ and non-DM participants were matched by gender in 7 studies, by age in 6 studies, by race distribution in 2, by socioeconomic status in 3 , by living in the same area in two, and by Tanner puberty states in another one. With regard to the type of flow rate 9 studies collected UWS, 4 SWS, 2 USP, one SSS, one USS, and one collected SSP.

Three studies did not explain the hour of collection of saliva and 4 studies did not specify the saliva collection duration. Two studies collected salivary flow during 10 minutes and 6 studies during 5 minutes. Five studies $[13,17,18,20,21]$ did not show or clarify correctly the statistical methods. Regarding quality assessment, only one study [13] obtained JBI scores $\geq 5$ (Table 3 ). Therefore, due to the poor quality of the majority of the included studies no meta-analysis was performed.

Only one study reflected prevalence of hyposalivation as outcome [7]. The definition of hyposalivation was UWS < $0.1 \mathrm{~mL} / \mathrm{min}$ and SWS $<0.5 \mathrm{~mL} / \mathrm{min}$ (actually $<0.7 \mathrm{~mL} / \mathrm{min}$ is considered). The study showed that DM patients had significantly greater hyposalivation prevalence than CG.

\subsection{Main Findings}

3.3.1. Prevalence of Xerostomia in the DM/CG Population. The prevalence of xerostomia was analyzed in 7 studies (Table 2). In adults T2DM xerostomia prevalence varied between $12.5 \%$ and $53.5 \%$, compared to $0-28.4 \%$ in the CG [7-10]. Only three studies [8-10] (two with the same study population $[9,10])$ showed that DM patients suffered significantly more xerostomia than non-DM patients. One study realized by Bernardi et al. [8] showed that PCDM patients suffered more xerostomia prevalence than WCDM patients, 54\% and 47\%, respectively.

There was only one study about xerostomia in adults NIDDM [11]. This study showed that prevalence of xerostomia in NIDDM patients is greater than in CG population, $50 \%$ versus $30 \%$, but this result was not significant.

Only one work was realized in children and adolescents T1DM between 10 and 19 years old. This study showed that prevalence of xerostomia was greater in T1DM patients than non-T1DM patients $(0 \%)$, and the prevalence was greater in PCDM patients (100\%) than WCDM patients $(80 \%)$.

\subsubsection{Quantity of Salivary Flow Rate in the DM/CG Popula-} tion. The quantity of salivary flow rate was analyzed in 12 studies (Table 3). There was only one study in adults TIDM [13]; this study showed that SWS flow rate was lower in $\mathrm{DM}$ versus non-DM patients, 1.30 versus $1.54 \mathrm{~mL} / \mathrm{min}$, and obtained higher salivary flow rate in PCDM than WCDM (1.31 versus $1.34 \mathrm{~mL} / \mathrm{min}$ ). The study did not show significant statistical results. In adults IDDM it was another study [22] that found significantly lower UWS flow rate in DM patients than non-DM patients, $0.35 \pm 0.24$ versus $0.48 \pm 0.23 \mathrm{~mL} / \mathrm{min}$.
A considerable part of studies were realized in adults T2DM [7, 8, 15-18]. Four of them evaluated UWS $[7,15,17$, 18]; the UWS flow rate in T2DM and non-T2DM patients varied between $0.16-0.5 \mathrm{~mL} / \mathrm{min}$ and $0.26-0.75 \mathrm{~mL} / \mathrm{min}$, respectively. Two of these studies [7,15] obtained greater significant UWS flow rate in T2DM than in CG patients. In addition, Chavez et al. [18] assessed the UWS flow rate in WCDM and PCDM adults T2DM; they found higher rates in PCDM than WCDM.

Three studies assessed SWS flow rate in T2DM $[7,8,16]$. The rates of SWS in T2DM and non-T2DM patients varied between $0.63-0.95 \mathrm{~mL} / \mathrm{min}$ and $1.14-1.95 \mathrm{~mL} / \mathrm{min}$, respectively. Two of them $[7,8]$ showed significant statistical results. The study of Bernardi et al. [8] showed that WCDM had greater SWS rates than PCDM.

USP flow rates were analyzed in two studies [17, 25]; only in one of them [17] did T2DM patients show lower rates than non-DM patients; none obtained significant results.

There were four studies $[12,19-21]$ that reported salivary flow rates in children and adolescents T1DM and IDDM between 4 and 19 years old. All studies evaluated UWS; the rates in $\mathrm{DM}$ population varied between 0.15 and $0.79 \mathrm{~mL} / \mathrm{min}$ and in non-DM patients 0.25 and $1.06 \mathrm{~mL} / \mathrm{min}$. Three studies $[12,19,20]$ obtained significant lower rates in T1DM and IDDM patients. Javed et al. [12] showed that WCDM had greater UWS rates than PCDM, but this result was nonsignificant.

3.3.3. Prevalence of Hyposalivation in the DM/CG Population. Only one study evaluated this outcome and showed that hyposalivation prevalence was significantly greater in T2DM versus CG patients, $45 \%$ versus $2.5 \%$.

\section{Discussion}

Multiple epidemiologic studies have suggested that xerostomia is frequent among DM patients. In addition, there are studies that have showed that DM patients presented lower salivary flow rates than non-DM population [26]. These salivary disorders could be associated with a poor quality of life and could increase the susceptibility to caries and oral infections in DM patients, particularly when there has been dehydration and inadequate blood glucose control [18]. DM is probably the most frequent metabolic disease with salivary implications, due to its high frequency. This systematic review was performed to analyze the prevalence of xerostomia and hyposalivation and the rates of salivary flow in DM patients in relation to non-DM patients. We specified explicit eligibility criteria, conducted comprehensive searches, and assessed risk of bias using criteria specific to this review.

4.1. Risk of Bias within Studies. Selection bias regarding the study population was minimized through the restriction to population-based studies. At the same time, we detected some sources of information bias. Firstly, the majority of studies $[7,9-13,15,16,20-22]$ do not specify the DM diagnosis. Secondly, most of the studies $[7,8,11,12,16,18,20-22]$ 
did not show the observation period and the type of recruitment of DM cases. With respect to the salivary flow rate, not all the studies reported the same type of salivary flow and the same technique, and these could also cause bias. Finally, DM and non-DM are not correctly matched; there are studies that did not even match age and gender $[8,12$, $15,19,20]$ and there is no study that matched correctly the use of drugs and illness (apart DM), so important in xerostomia/hyposalivation etiology. As we can see in Tables 2 and 3, the sample size in the majority of studies was small (especially in adults T2DM), considering that DM is a very frequent disease. With respect to the statistical analysis, not all the studies reported continuous variables in mean \pm standard deviation.

4.2. Risk of Bias across Studies. Due to the fact that only articles published in the English language were reviewed, publication (language) bias could not be ruled out. Although we searched three databases, we cannot guarantee that some related papers might not have been identified. However, we did check the reference lists of reviewed articles to identify relevant studies. The studies reviewed presented different types of DM and DM and non-DM patients of different age (see Section 2) that could cause detection bias. We minimized it by grouping together studies with similar age and the same DM type in every outcome.

4.3. Main Findings. We identified 15 studies reporting prevalence of xerostomia/hyposalivation and rates of salivary flow in DM population. Comparisons between studies were limited due to different types of DM, different types of salivary flow, and heterogeneous demographic characteristics (age, ethnic origin) of the studied individual. In addition, the quality assessment of studies was low. Hence, no quantitative data synthesis was performed. Nevertheless, there are some patterns that can be described.

4.3.1. Xerostomia Prevalence. All studies about this outcome showed higher prevalence of xerostomia in DM patients in relation to non-DM population, $12.5 \%-53.5 \%$ compared to $0-30 \%[7-12,18]$. Nevertheless, only four studies $[8-10,12]$ (two with the same study population $[9,10]$ ) have shown significant statistical results. Two studies $[8,12]$ showed that WCDM patients have lower xerostomia prevalence than PCDM.

4.3.2. Salivary Flow Rates. All studies [7, 12, 15, 17-22] that analyzed the quantity of UWS in DM population in relation to non-DM patients reported higher UWS rates in non$\mathrm{DM}$ than in DM patients. The variation flow rate among the different studies in each group (DM/CG) is very large. Six $[7,12,15,19,20,22]$ of these studies showed significant statistical results. The large variation flow rate among the studies could be due to the different criteria used to measure UWS. The time of measurement strongly influences the flow rate, so the saliva test (not only UWS) has to be performed at a fixed time-point of a limited time interval early morning due to the circadian rhythm of salivary flow $[4,27]$. In addition, the duration of salivary collection is also important [4], and not all studies reflected the same duration. In the studies, where the time of flow rate collection is present, this time varied between 5 and 10 minutes. In addition, it is not clear if WCDM patients have higher UWS rates than PCDM; of two studies $[12,18]$ discussing this topic only one [12] showed nonsignificant higher rates for WCDM patients.

The comparison of the SWS rates between DM and nonDM patients showed that rates were higher in non-DM patients $[7,8,13,16]$, but only half of the studies showed significant statistical results $[7,8]$. The SWS flow rate varies very much among the different studies, in the manner of UWS; the possible reason was specified previously.

4.3.3. Hyposalivation Prevalence. Only one study [7] was about hyposalivation; this study showed significant statistical higher hyposalivation prevalence in DM than non-DM patients (45\% versus $2.5 \%$ ). The hyposalivation SWS level in this study is not actually accepted $(<0.7 \mathrm{~mL} / \mathrm{min})$ if not $<0.5 \mathrm{~mL} / \mathrm{min}$; therefore, the results could be biased.

4.4. Strengths and Limitations. The selection of studies for this systematic review was based on a systematic search approach with clearly determined search strategies. We included only those studies reporting xerostomia prevalence/salivary flow rate/hyposalivation within the DM population in relation to a non-DM control group. Moreover, we analyzed these outcomes in separate groups according to age and type of DM. This approach allows limited comparison of the studies despite a high degree of heterogeneity. Our review also has some limitations. Although three databases were searched, we cannot rule out having missed relevant studies, also due to publication bias. The studies published in languages other than English were not included. Most studies reporting our outcomes were conducted in economically developed areas such as USA and Sweden and thus do not represent a worldwide perspective.

In addition, there are studies previous to the year 2000. The change in the diagnostic criteria for DM from $140 \mathrm{mg} / \mathrm{dL}$ $(7.8 \mathrm{mmol} / \mathrm{L})$ to $126 \mathrm{mg} / \mathrm{dL}(7.0 \mathrm{mmol} / \mathrm{L})$ in the fasting plasma glucose level in 1997 [28] led to an increase of the diabetic population due to the inclusion of less severe stages of the disease, and this must be taken into consideration when interpreting the results. Criteria for the diagnosis of prediabetes and DM could change periodically [2]; therefore, it is very important to realize the studies according to the current criteria.

\section{Conclusions}

The review conducted demonstrated the considerable variation in prevalence of xerostomia and salivary flow rates among DM population in relation to non-DM patients. Most studies found a higher prevalence of xerostomia and lower salivary flow rates in DM with respect to CG. We found only a study about hyposalivation that showed higher prevalence in DM than non-DM patients. A few studies showed that WCDM patients have lower xerostomia prevalence and 
higher salivary flow rates than PCDM patients. Owing to the high degree of heterogeneity regarding the types of DM, diagnosis of DM, age of patients, and types and techniques of salivary flow collection, it was difficult to compare the studies. In addition, the quality assessment showed the low quality of the existing studies. Therefore, the results of this systematic review were inconsistent.

We recommend that new studies analyzing the xerostomia and salivary flow rate in the DM population should use more precise and current definitions concerning the determination and diagnosis of DM patients and salivary flow rate collection. New studies should match correctly DM and non-DM patients, keeping in mind xerostomia associated drugs and illness (other than DM). New studies are required that consider hyposalivation in DM patients because a reduction in salivary flow is not always pathological.

\section{Appendix}

\section{List of Excluded Studies and Reason of Exclusion}

[1] F. Javed, HB. Ahmed, A. Mehmood, A. Saeed, K. Al-Hezaimi, and LP. Samaranayake, "Association between glycemic status and oral Candida carriage in patients with prediabetes", Oral surgery, oral medicine, oral pathology and oral radiology, vol. 117, no. 1, pp. 53201358, 2014. (The outcomes were not present.)

[2] E. de la Rosa-Garcia, M. Miramontes-Zapata, LO. Sanchez-Vargas, and A. Mondragon-Padilla, "Oral colonisation and infection by Candida sp. in diabetic and nondiabetic patients with chronic kidney disease on dialysis", Nefrologia, vol. 33, no. 6, pp. 764-770, 2013. (Study about oral candidiasis in DM and non-DM patients.)

[3] DH. Han, MS. Kim, HS. Shin, KP. Park, and HD. Kim, "Association between periodontitis and salivary nitric oxide metabolites among community elderly Koreans", Journal of Periodontology, vol. 84, no. 6, pp. 776-784, 2013. (Study not performed in DM patients. Study about periodontitis.)

[4] G. Teratani, S. Awano, I. Soh, A. Yoshida, N. Kinoshita, T. Hamasaki et al., "Oral health in patients on haemodialysis for diabetic nephropathy and chronic glomerulonephritis", Clinical oral investigations, vol. 17, no. 2, pp. 483-489, 2013. (Study about oral health in haemodialysis patients.)

[5] M. Vesterinen, H. Ruokonen, J. Furuholm, E. Honkanen, and JH. Meurman, "Clinical questionnaire study of oral health care and symptoms in diabetic vs. non-diabetic predialysis chronic kidney disease patients", Clinical oral investigations, vol. 16, no. 2, pp. 559-563, 2012. (Kidney disease patients.)

[6] P. Vestergaard, K. Schwartz, L. Rejnmark, L. Mosekilde, and EM. Pinholt, "Oral bisphosphonate use increases the risk for inflammatory jaw disease: a cohort study", Journal of oral and maxillofacial surgery, vol. 70, no. 4, pp. 821-829, 2012. (This study does not evaluate the outcomes. No DM patients.)

[7] J. Fricton, DB. Rindal, W. Rush, T. Flottemesch, G. Vazquez, MJ. Thoele et al., "The effect of electronic health records on the use of clinical care guidelines for patients with medically complex conditions", Journal of the American Dental Association, vol. 142, no. 10, pp. 1133-1142, 2011. (This study does not evaluate the outcomes. No DM patients.)

[8] AMH. Syrjala, L. Raatikainen, K. Komulainen, M. Knuuttila, P. Ruoppi, S. Hartikainen et al., "Salivary flow rate and periodontal infection - a study among subjects aged 75 years or older", Oral diseases, vol. 17, no. 4, pp. 387-392, 2011. (No DM patients.)

[9] AMH. Syrjala, P. Ylostalo, S. Hartikainen, R. Sulkava, and M. Knuuttila, "Number of teeth and selected cardiovascular risk factors among elderly people", Gerodontology, vol. 27, no. 3, pp. 189-192, 2010. (No DM patients.)

[10] E. de la Rosa Garcia, A. Mondragon Padilla, S. Aranda Romo, and MA. Bustamante Ramirez, "Oral mucosa symptoms, signs and lesions, in end-stage renal disease and non-end-stage renal disease diabetic patients", Medicina oral, patologia oral y cirugia bucal, vol. 11, no. 6, pp. E467-473, 2006. (End-stage renal disease DM patients.)

[11] JM. Sung, SC. Kuo, HR. Guo, SF. Chuang, SY. Lee, and JJ. Huang, "The role of oral dryness in interdialytic weight gain by diabetic and non-diabetic haemodialysis patients", Nephrology, dialysis, transplantation, vol. 21, no. 9, pp. 25212528, 2006. (DM and non-DM haemodialysis patients.)

[12] AA. Alavi, E. Amirhakimi, and B. Karami, "The prevalence of dental caries in 5-18-year-old insulindependent diabetics of Fars Province, southern Iran", Archives of Iranian medicine, vol. 9, no. 3, pp. 254-260, 2006. (Does not evaluate outcomes.)

[13] HW. Boyce, and MR. Bakheet, "Sialorrhea: a review of a vexing, often unrecognized sign of oropharyngeal and esophageal disease", Journal of clinical gastroenterology, vol. 39, no. 2, pp. 89-97, 2005. (Does not evaluate outcomes.)

[14] GE. Sandberg, and KF. Wikblad, "Oral health and health-related quality of life in type 2 diabetic patients and non-diabetic controls", Acta odontologica Scandinavica, vol. 61, no. 3, pp. 141-148, 2003. (Does not evaluate outcomes.)

[15] JS. Mattson JS, and DR. Cerutis, "Diabetes mellitus: a review of the literature and dental implications", Compendium of continuing education in dentistry, vol. 22, no. 9, pp. 757-760, 2001. (Review of the literature.)

[16] CH. Kao, SC. Tsai, and SS. Sun, "Scintigraphic evidence of poor salivary function in type 2 diabetes", Diabetes care, vol. 24, no. 5, pp. 952-953, 2001. (Does not evaluate outcomes.)

[17] EM. Chavez, LN. Borrell, GW. Taylor, and JA. Ship, "A longitudinal analysis of salivary flow in control subjects and older adults with type 2 diabetes", Oral surgery, oral medicine, oral pathology, oral radiology, and endodontics, vol. 91, no. 2, pp. 166-173, 2001. (Longitudinal study.)

[18] AL. Mason, L. Xu, L. Guo, and RF. Garry, "Retroviruses in autoimmune liver disease: genetic or environmental agents?", Archivum immunologiae et therapiae experimentalis, vol. 47, no. 5, pp. 289-297, 1999. (Does not evaluate outcomes. No DM patients.)

[19] RE. Persson, GR. Persson, HA. Kiyak, and LV. Powell, "Oral health and medical status in dentate low-income older persons", Special care in dentistry, vol. 18, no. 2, pp. 70-77, 1998. (Does not evaluate outcomes.) 
[20] CP. Robinson, S. Yamachika, CE. Alford, C. Cooper, EL. Pichardo, N. Shah et al., "Elevated levels of cysteine protease activity in saliva and salivary glands of the nonobese diabetic (NOD) mouse model for Sjogren syndrome", Proceedings of the National Academy of Sciences of the United States of America, vol. 94, no. 11, pp. 5767-5771, 1997. (Animal study. Does not evaluate outcomes.)

[21] MR. Quirino, EG. Birman, and CR. Paula, "Oral manifestations of diabetes mellitus in controlled and uncontrolled patients", Brazilian dental journal, vol. 6, no. 2, pp. 131136, 1995. (Does not evaluate outcomes.)

[22] K. Kimura, K. Ezoe, H. Yokozeki, I. Katayama, and K. Nishioka, "Elevated serum CA125 in progressive systemic sclerosis with pleural effusion", The Journal of dermatology, vol. 22, no. 1, pp. 28-31, 1995. (Does not evaluate outcomes. No DM patients.)

[23] P. Collin, T. Reunala, E. Pukkala, P. Laippala, O. Keyrilainen, and A. Pasternack, "Coeliac disease-associated disorders and survival”, Gut, vol. 35, no. 9, pp. 1215-1218, 1994. (Does not evaluate outcomes. No DM patients.)

[24] M. Mogi, M. Harada, T. Kage, T. Chino, and K. Yoshitake, "Two-dimensional electrophoresis of human salivary proteins from patients with sialoadenopathy", Archives of oral biology, vol. 38, no. 12, pp. 1135-1139, 1993. (Does not evaluate outcomes. No DM patients.)

[25] LM. Sreebny, A. Yu, A. Green, and A. Valdini, "Xerostomia in diabetes mellitus", Diabetes care, vol. 15, no. 7, pp. 900-904, 1992. (Type of DM not specified.)

[26] L. Tabak, ID. Mandel, D. Karlan, and H. Baurmash, "Alterations in lactoferrin in salivary gland disease", Journal of dental research, vol. 57, no. 1, pp. 43-47, 1978. (Does not evaluate outcomes. No DM patients.)

[27] S. Conner, B. Iranpour, and J. Mills, “Alteration in parotid salivary flow in diabetes mellitus", Oral surgery, oral medicine, and oral pathology, vol. 30, no.1, pp. 55-59, 1970. (No control group.)

[28] M. Tremblay, D. Brisson, and D. Gaudet, "Association between salivary $\mathrm{pH}$ and metabolic syndrome in women: a cross-sectional study", BMC oral health, vol. 12, pp. 40, 2012. (Does not evaluate outcomes. No DM patients.)

[29] AT. Eltas, M. Keles, and V. Canakci, "Assessment of oral health in peritoneal dialysis patients with and without diabetes mellitus", Peritoneal dialysis international, vol. 32, no. 1, pp. 81-85, 2012. (Dialysis patients.)

[30] M. Isola, P. Solinas, E. Proto, M. Cossu, and MS. Lantini, "Reduced statherin reactivity of human submandibular gland in diabetes", Oral diseases, vol. 17, no. 2, pp. 217-220, 2011. (Does not evaluate outcomes.)

[31] P. Gumus, N. Buduneli, S. Cetinkalp, SI. Hawkins, D. Renaud, DF. Kinane DF et al., "Salivary antioxidants in patients with type 1 or 2 diabetes and inflammatory periodontal disease: a case-control study", Journal of periodontology, vol. 80, no.9, pp. 1440-1446, 2009. (Does not evaluate outcomes.)

[32] J. Siudikiene, V. Machiulskiene, B. Nyvad, J. Tenovuo, and I. Nedzelskiene, "Dental caries increments and related factors in children with type 1 diabetes mellitus", Caries research, vol. 42, no. 5, pp. 354-362, 2008. (Longitudinal study.)
[33] S. Aydin, "A comparison of ghrelin, glucose, alphaamylase and protein levels in saliva from diabetics", Journal of biochemistry and molecular biology, vol. 40, no. 1, pp. 29-35, 2007. (Does not evaluate outcomes.)

[34] KM. Karjalainen, ML. Knuuttila, and ML. Kaar, "Salivary factors in children and adolescents with insulindependent diabetes mellitus", Pediatric dentistry, vol. 18, no. 4, pp. 306-311, 1996. (Control group not present.)

[35] P. Canepari, N. Zerman, and G. Cavalleri, "Lack of correlation between salivary Streptococcus mutans and lactobacilli counts and caries in IDDM children", Minerva stomatologica, vol. 43, no. 11, pp. 501-505, 1994. (Flow rate dependent on caries of DM/non-DM patients.)

[36] M. Sutters, C. Brace, E. Hatfield, A. Whitehurst, SL. Lightman, and WS. Peart, "Control of sodium excretion in patients with cranial diabetes insipidus maintained on desamino- 8-D-arginine vasopressin", Clinical science, vol. 85, no. 5, pp. 599-606, 1993. (Does not evaluate outcomes.)

[37] PJ. Lamey, BM. Fisher, and BM. Frier, "The effects of diabetes and autonomic neuropathy on parotid salivary flow in man", Diabetic medicine, vol. 3, no. 6, pp. 537-540, 1986. (Results in relation to autonomic neuropathy.)

[38] M. May, R. Wette, WB. Hardin, and J. Sullivan, “The use of steroids in Bell's palsy: a prospective controlled study", The Laryngoscope, vol. 86, no. 8, pp. 1111-1122, 1976. (No DM patients.)

\section{Competing Interests}

The authors declare that there is no conflict of interests regarding the publication of this paper.

\section{Authors' Contributions}

Rosa María López-Pintor conceived and designed the experiments. Rosa María López-Pintor, Elisabeth Casañas, José González-Serrano, Julia Serrano, and Lucía Ramírez performed the experiments. Rosa María López-Pintor, Elisabeth Casañas, José González-Serrano, Julia Serrano, Lucía Ramírez, and Gonzalo Hernández analyzed the data. Lorenzo de Arriba contributed reagents/materials/analysis tools. Rosa María López-Pintor wrote the paper. Gonzalo Hernández contributed to the concept, design, and drafting of the protocol. Rosa María López-Pintor, Elisabeth Casañas participated in the development of the systematic search strategies. Gonzalo Hernández, Lorenzo de Arriba made major contributions to the write-up and editing of systematic review. Gonzalo Hernández, Lorenzo de Arriba, and Elisabeth Casañas critically revised the paper for important intellectual content and approved the final version.

\section{References}

[1] IDF Diabetes Atlas-seventh edition 2015, http://www diabetesatlas.org.

[2] J. J. Chamberlain, A. S. Rhinehart, C. F. Shaefer, and A. Neuman, "Diagnosis and management of diabetes: synopsis of the 2016 american diabetes association standards of medical care in 
diabetes," Annals of Internal Medicine, vol. 164, no. 8, pp. 542$552,2016$.

[3] C. Scully and D. H. Felix, "Oral medicine-update for the dental practitioner: dry mouth and disorders of salivation," British Dental Journal, vol. 199, no. 7, pp. 423-427, 2005.

[4] C. D. Löfgren, C. Wickström, M. Sonesson, P. T. Lagunas, and C. Christersson, "A systematic review of methods to diagnose oral dryness and salivary gland function," BMC Oral Health, vol. 12, no. 1, article 29, 2012.

[5] J. Saleh, M. A. Z. Figueiredo, K. Cherubini, and F. G. Salum, "Salivary hypofunction: an update on aetiology, diagnosis and therapeutics," Archives of Oral Biology, vol. 60, no. 2, pp. 242255, 2014.

[6] P. Han, P. Suarez-Durall, and R. Mulligan, "Dry mouth: a critical topic for older adult patients," Journal of Prosthodontic Research, vol. 59, no. 1, pp. 6-19, 2015.

[7] A. C. U. Vasconcelos, M. S. M. Soares, P. C. Almeida, and T. C. Soares, "Comparative study of the concentration of salivary and blood glucose in type 2 diabetic patients," Journal of Oral Science, vol. 52, no. 2, pp. 293-298, 2010.

[8] M. J. Bernardi, A. Reis, A. D. Loguercio, R. Kehrig, M. F. Leite, and J. Nicolau, "Study of the buffering capacity, $\mathrm{pH}$ and salivary flow rate in type 2 well-controlled and poorly controlled diabetic patients," Oral Health \& Preventive Dentistry, vol. 5, no. 1, pp. 73-78, 2007.

[9] G. E. Sandberg, H. E. Sundberg, C. A. Fjellstrom, and K. F. Wikblad, "Type 2 diabetes and oral health: a comparison between diabetic and non- diabetic subjects," Diabetes Research and Clinical Practice, vol. 50, no. 1, pp. 27-34, 2000.

[10] G. E. Sandberg, H. E. Sundberg, and K. F. Wikblad, "A controlled study of oral self-care and self-perceived oral health in type 2 diabetic patients," Acta Odontologica Scandinavica, vol. 59, no. 1, pp. 28-33, 2001.

[11] M. B. Zielinski, D. Fedele, L. J. Forman, and S. C. Pomerantz, "Oral health in the elderly with non-insulin-dependent diabetes mellitus," Special Care in Dentistry, vol. 22, no. 3, pp. 94-98, 2002.

[12] F. Javed, U. Sundin, M. Altamash, B. Klinge, and P.-E. EngstrÖm, "Self-perceived oral health and salivary proteins in children with type 1 diabetes," Journal of Oral Rehabilitation, vol. 36, no. 1, pp. 39-44, 2009.

[13] E. Edblad, S.-Å. Lundin, B. Sjödin, and J. Åman, "Caries and salivary status in young adults with type 1 diabetes," Swedish Dental Journal, vol. 25, no. 2, pp. 53-60, 2001.

[14] C. P. Bots, H. S. Brand, E. C. I. Veerman et al., "Interdialytic weight gain in patients on hemodialysis is associated with dry mouth and thirst," Kidney International, vol. 66, no. 4, pp. 16621668, 2004.

[15] T. J. Lasisi and A. A. Fasanmade, "Salivary flow and composition in diabetic and non-diabetic subjects," Nigerian Journal of Physiological Sciences, vol. 27, no. 1, pp. 79-82, 2012.

[16] D. C. de Lima, G. C. Nakata, I. Balducci, and J. D. Almeida, "Oral manifestations of diabetes mellitus in complete denture wearers," The Journal of Prosthetic Dentistry, vol. 99, no. 1, pp. 60-65, 2008.

[17] M. W. J. Dodds, C.-K. Yeh, and D. A. Johnson, "Salivary alterations in type 2 (non-insulin-dependent) diabetes mellitus and hypertension," Community Dentistry and Oral Epidemiology, vol. 28, no. 5, pp. 373-381, 2000.

[18] E. M. Chavez, G. W. Taylor, L. N. Borrell, and J. A. Ship, "Salivary function and glycemic control in older persons with diabetes,"
Oral Surgery, Oral Medicine, Oral Pathology, Oral Radiology, and Endodontics, vol. 89, no. 3, pp. 305-311, 2000.

[19] C. Alves, R. Menezes, and M. Brandão, "Salivary flow and dental caries in Brazilian youth with type 1 diabetes mellitus," Indian Journal of Dental Research, vol. 23, no. 6, pp. 758-762, 2012.

[20] M. E. López, M. E. Colloca, R. G. Páez, J. N. Schallmach, M. A. Koss, and A. Chervonagura, "Salivary characteristics of diabetic children," Brazilian Dental Journal, vol. 14, no. 1, pp. 26-31, 2003.

[21] M. A. Belazi, A. Galli-Tsinopoulou, D. Drakoulakos, A. Fleva, and P. H. Papanayiotou, "Salivary alterations in insulindependent diabetes mellitus," International Journal of Paediatric Dentistry, vol. 8, no. 1, pp. 29-33, 1998.

[22] H. Ben-Aryeh, M. Cohen, Y. Kanter, R. Szargel, and D. Laufer, "Salivary composition in diabetic patients," The Journal of Diabetic Complications, vol. 2, no. 2, pp. 96-99, 1988.

[23] D. Moher, A. Liberati, J. Tetzlaff, and D. Altman, "Preferred reporting items for systematic reviews and meta-analyses: the PRISMA statement," PLoS Medicine, vol. 6, no. 7, Article ID e1000097, 2009.

[24] The Joanna Briggs Institute, The Joanna Briggs Institute Reviewers' Manual, The Joanna Briggs Institute, Adelaide, Australia, 2014.

[25] E. M. Chávez, L. N. Borrell, G. W. Taylor, and J. A. Ship, "A longitudinal analysis of salivary flow in control subjects and older adults with type 2 diabetes," Oral Surgery, Oral Medicine, Oral Pathology, Oral Radiology, and Endodontics, vol. 91, no. 2, pp. 166-173, 2001.

[26] L. M. Sreebny, A. Yu, A. Green, and A. Valdini, "Xerostomia in diabetes mellitus," Diabetes Care, vol. 15, no. 7, pp. 900-911, 1992.

[27] H. Flink, Å. Tegelberg, and F. Lagerlöf, "Influence of the time of measurement of unstimulated human whole saliva on the diagnosis of hyposalivation," Archives of Oral Biology, vol. 50, no. 6, pp. 553-559, 2005.

[28] K. G. M. M. Alberti and P. Z. Zimmet, "Definition, diagnosis and classification of diabetes mellitus and its complications. Part 1: diagnosis and classification of diabetes mellitus. Provisional report of a WHO consultation," Diabetic Medicine, vol. 15, no. 7, pp. 539-553, 1998. 


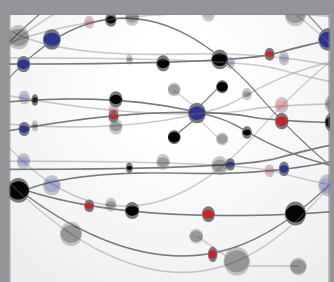

The Scientific World Journal
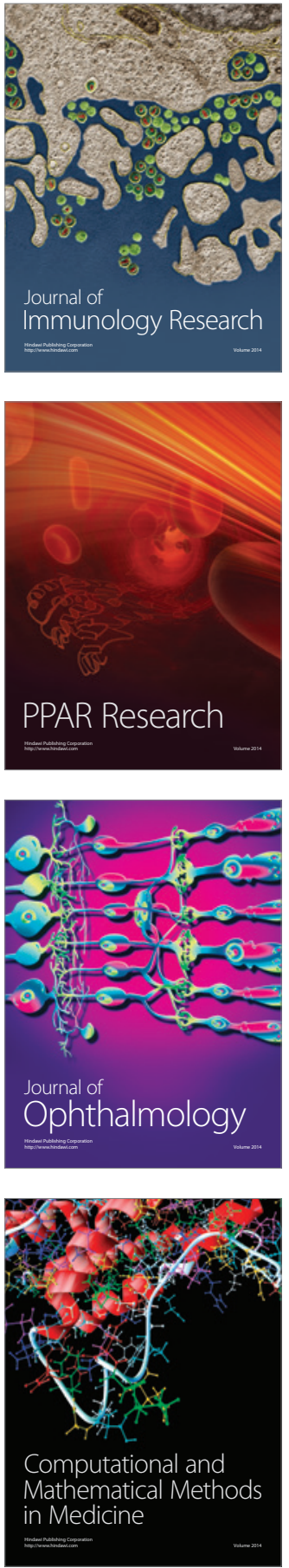

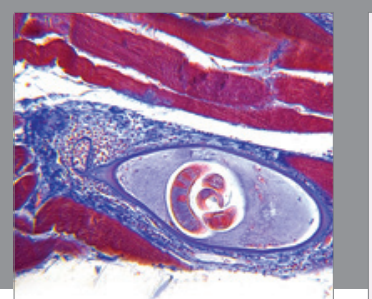

Gastroenterology Research and Practice

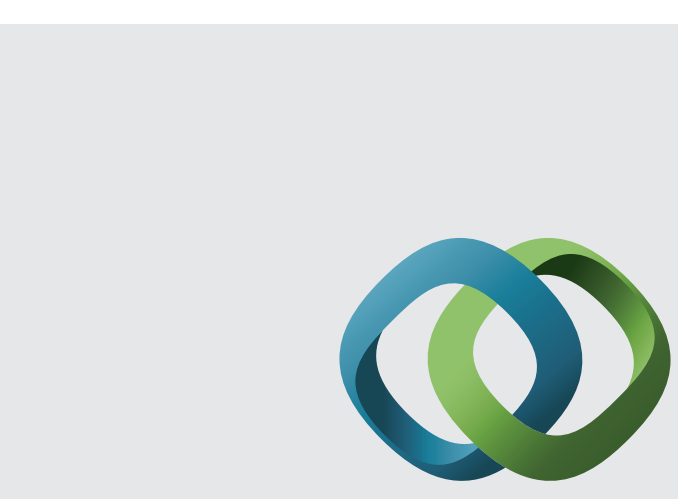

\section{Hindawi}

Submit your manuscripts at

http://www.hindawi.com
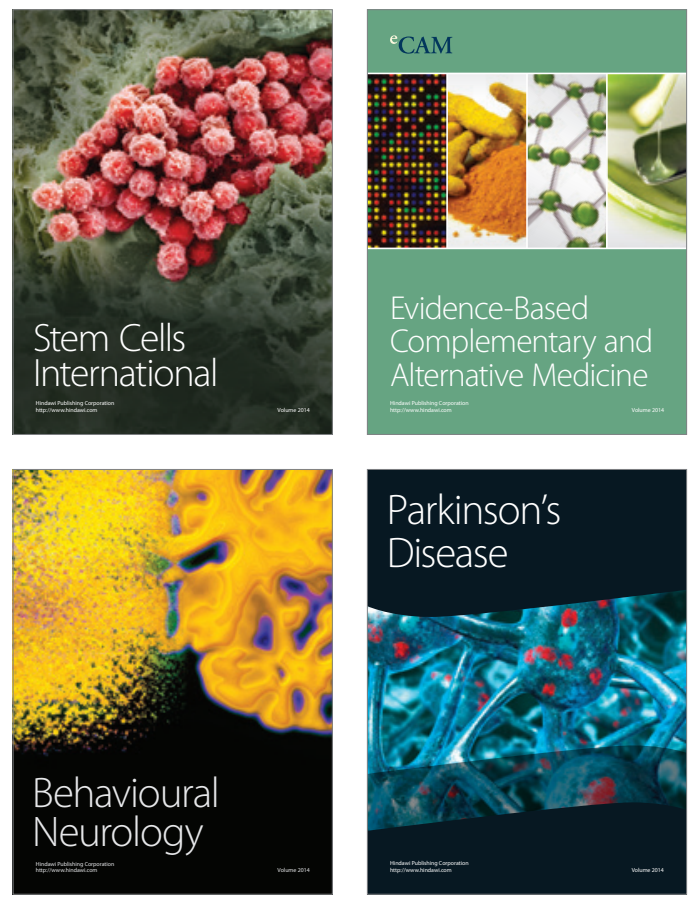
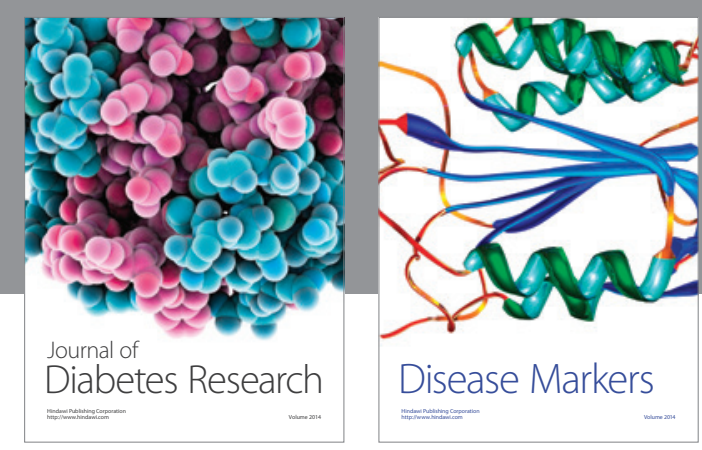

Disease Markers
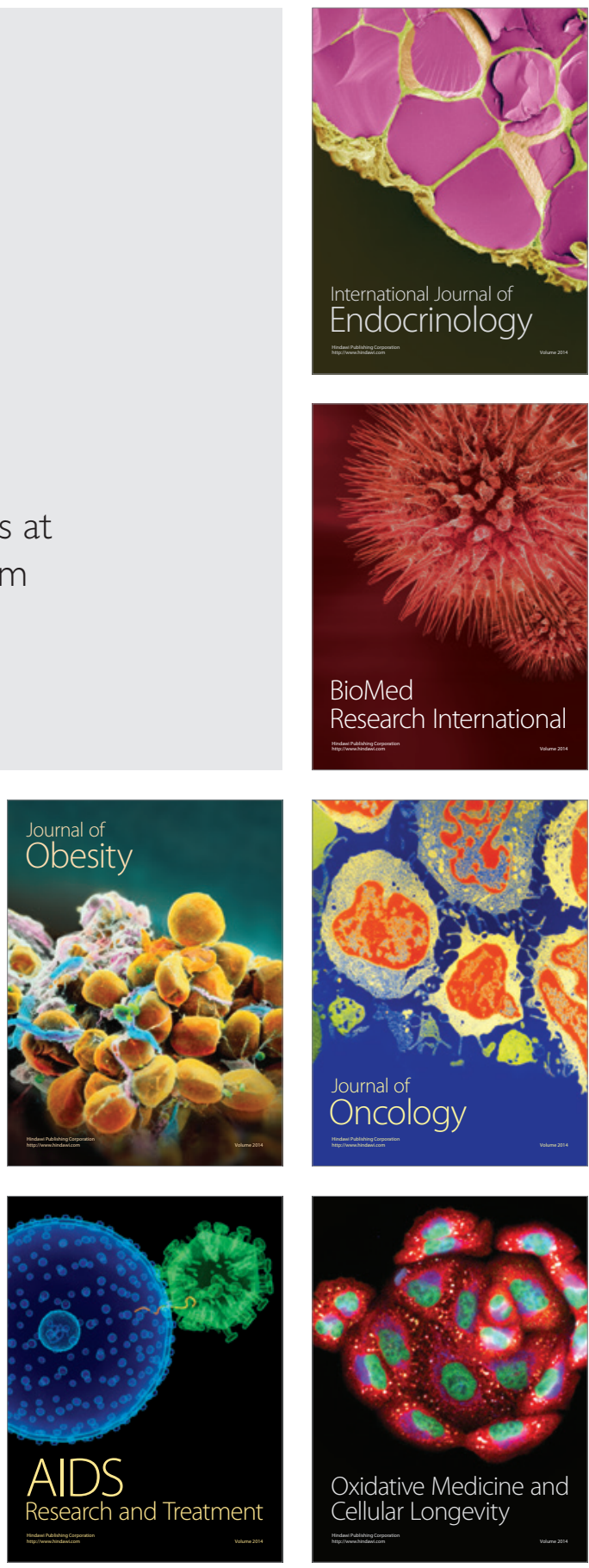